\title{
August 2013 Critical Care Case of the Month: My, That's a Big One
}

\author{
Andrew Waas, M.D. \\ Pulmonary Sciences and Critical Care Medicine \\ University of Colorado Hospital \\ Denver, Co
}

History of Present IIIness

A 75 year old male presented to the emergency department with complaints of three days of increasing nausea, generalized weakness, and dyspnea on exertion. He had undergone a radical prostatectomy 13 days prior to presentation from which he was recovering well until the onset of these symptoms. There was no associated chest pain, cough, fevers, chills or weight loss.

$\mathrm{PMH}, \mathrm{SH}, \mathrm{FH}$

He had a history of hypertension and prostate cancer for which he underwent a recent prostatectomy.

He was born in Colorado and had not traveled recently. There was no history of tobacco use, he drank ethanol on rare occasions, and did not use any illicit drugs.

There was no family history of illnesses of which he was aware.

Medications

Dutasteride $0.5 \mathrm{mg}$ daily

Telmisartan $40 \mathrm{mg}$ daily

Physical Exam

Blood pressure $142 / 85$, heart rate 108 , temperature $36.7 \mathrm{C}$, respiratory rate 25 , saturating $95 \%$ on $2 \mathrm{~L}$ oxygen.

Generally, he was in no distress, but was slightly tachypneic. Lungs were clear to auscultation bilaterally and he was tachycardic but regular. Otherwise, his exam was normal.

Laboratory

Laboratory evaluation revealed a mild leukocytosis at $13 \times 10^{6} \mathrm{cells} / \mathrm{mcL}$ with $72 \%$ neutrophils and 20\% lymphocytes. His basic metabolic panel (including creatinine) was normal; his liver function tests were likewise normal. 
Chest Radiography

His initial portable chest $\mathrm{x}$-ray is shown in Figure 1.

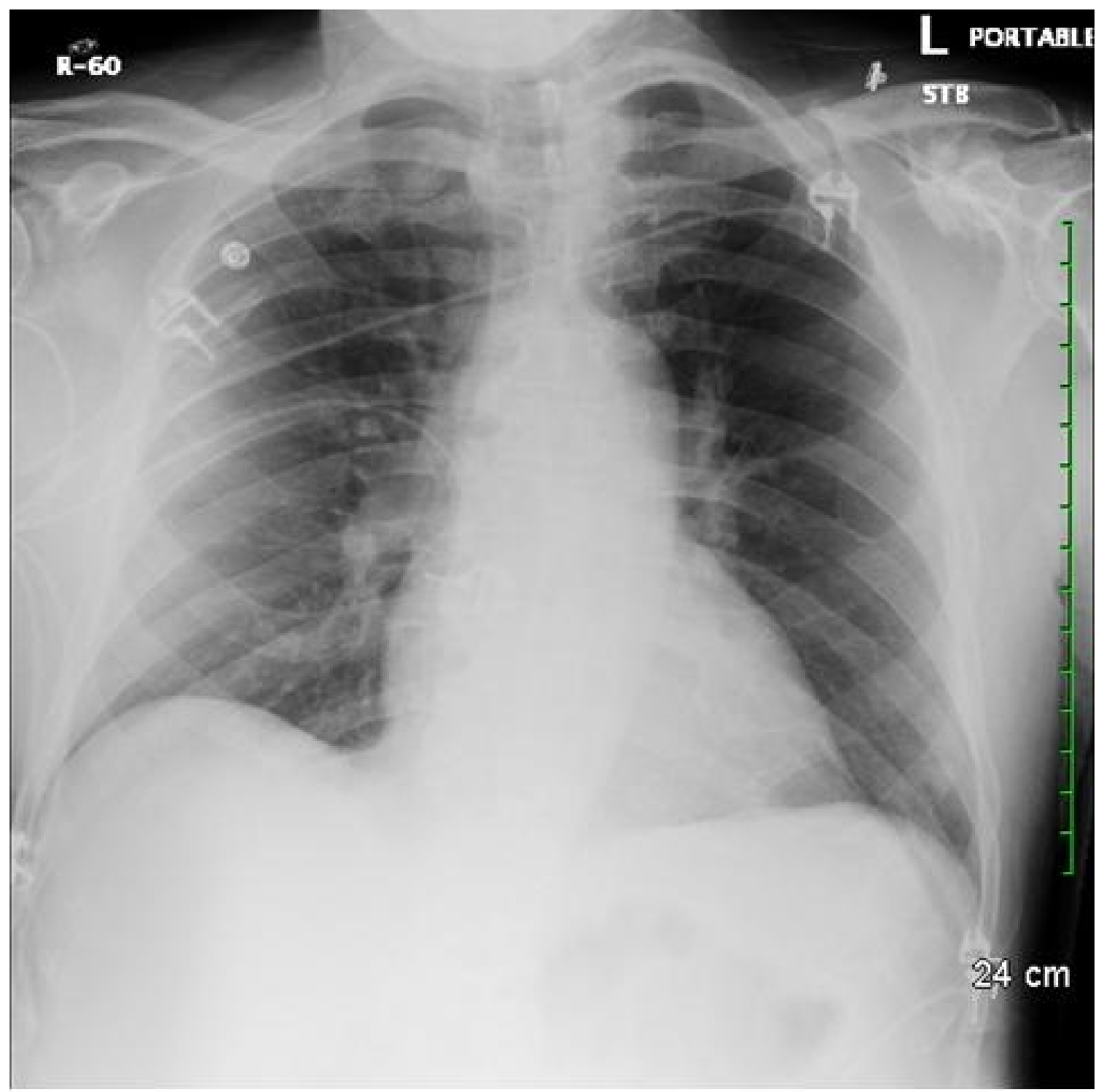

Figure 1. Initial portable chest x-ray

Which of the following best describes the chest $\mathrm{x}$-ray?

1. Cardiomegaly

2. Cavitating lung mass

3. Multifocal infiltrates

4. All of the above

5. None of the above 


\section{Correct! \\ 5. None of the above}

This is an essentially normal portable chest $\mathrm{x}$-ray. There are no focal infiltrates or evidence of cardiomegaly. Although the heart/chest ratio approaches 1:2 the heart appears somewhat enlarged because the chest $\mathrm{x}$-ray is a portable taken anterior to posterior.

Based on this initial evaluation, which is the next best step in management?

1. Immediately begin antibiotic coverage for health care associated pneumonia

2. Immediately begin antibiotic coverage for community acquired pneumonia

3. Obtain CT angiogram of the chest

4. Obtain high resolution $\mathrm{CT}$ of the chest

5. Perform bronchoscopy with bronchoalveolar lavage 


\section{Correct! \\ 3. Obtain CT angiogram of the chest}

The patient is requiring $2 \mathrm{~L} / \mathrm{min}$ of oxygen to maintain saturations, dyspneic, tachycardic, has a normal chest $\mathrm{x}$-ray and a recent prostatectomy. This should suggest a diagnosis. A CT angiogram of the chest is the procedure of choice to pursue this diagnosis and is shown in Figure 2.
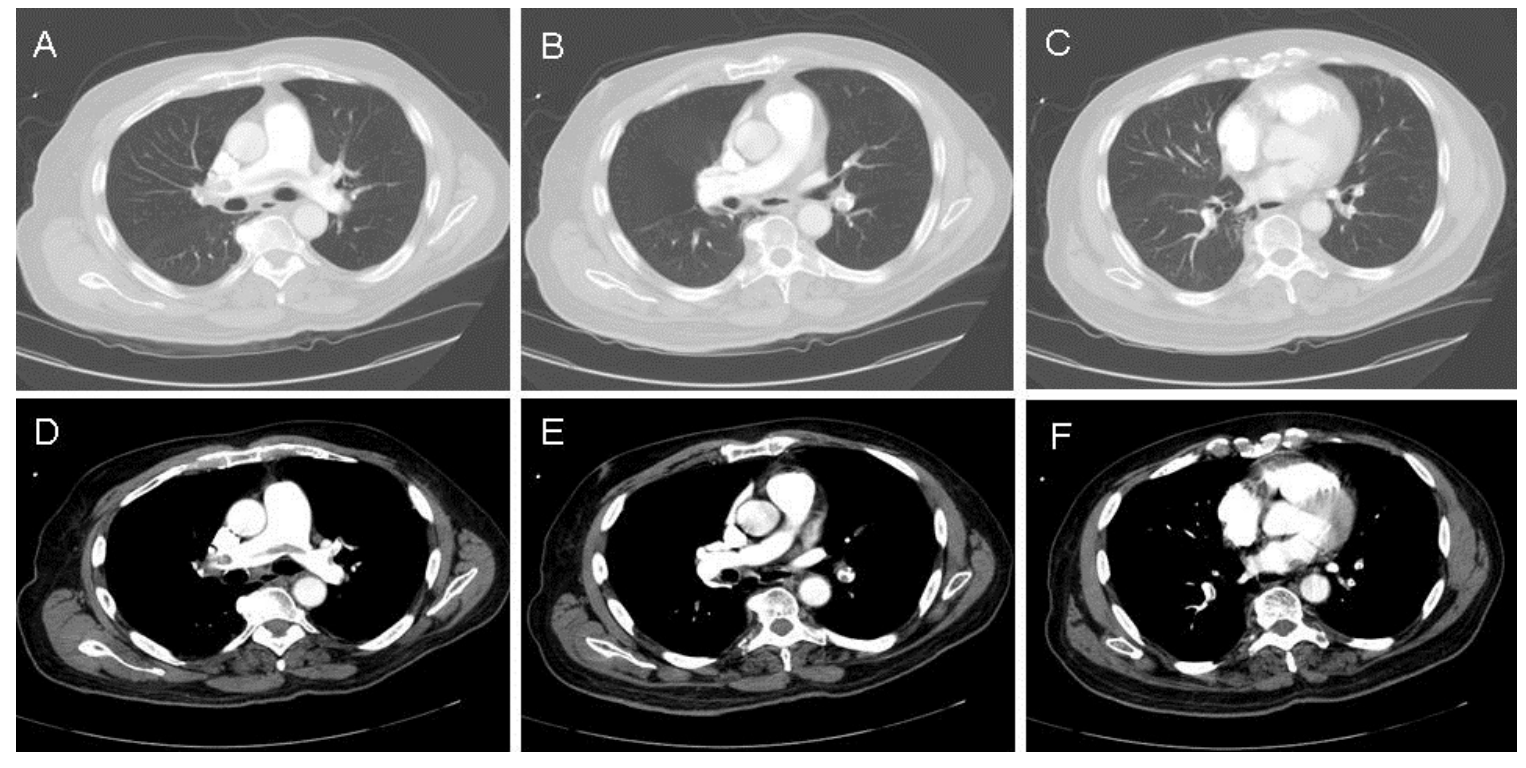

Figure 2. Selected static images from the CT angiogram showing lung windows $(A-C)$ and soft tissue windows (D-F).

Which of the following statements best describes the CT findings?

1. Bilateral basal ground-glass opacity and reticulation, suggesting noninfectious alveolitis with fibrosis

2. Bilateral basal low attenuation consistent with pulmonary parenchymal fat

3. Massive pulmonary embolus

4. Non-specific bilateral basal consolidation and adds little to what is already known from chest radiography

5. Patent ductus arteriosus 


\section{Correct! \\ 3. Massive pulmonary embolus}

The CT angiogram shows a massive pulmonary embolus as evidenced by pulmonary artery intraluminal filling defects. This is seen in both the lung and soft tissue windows (Figure 3).
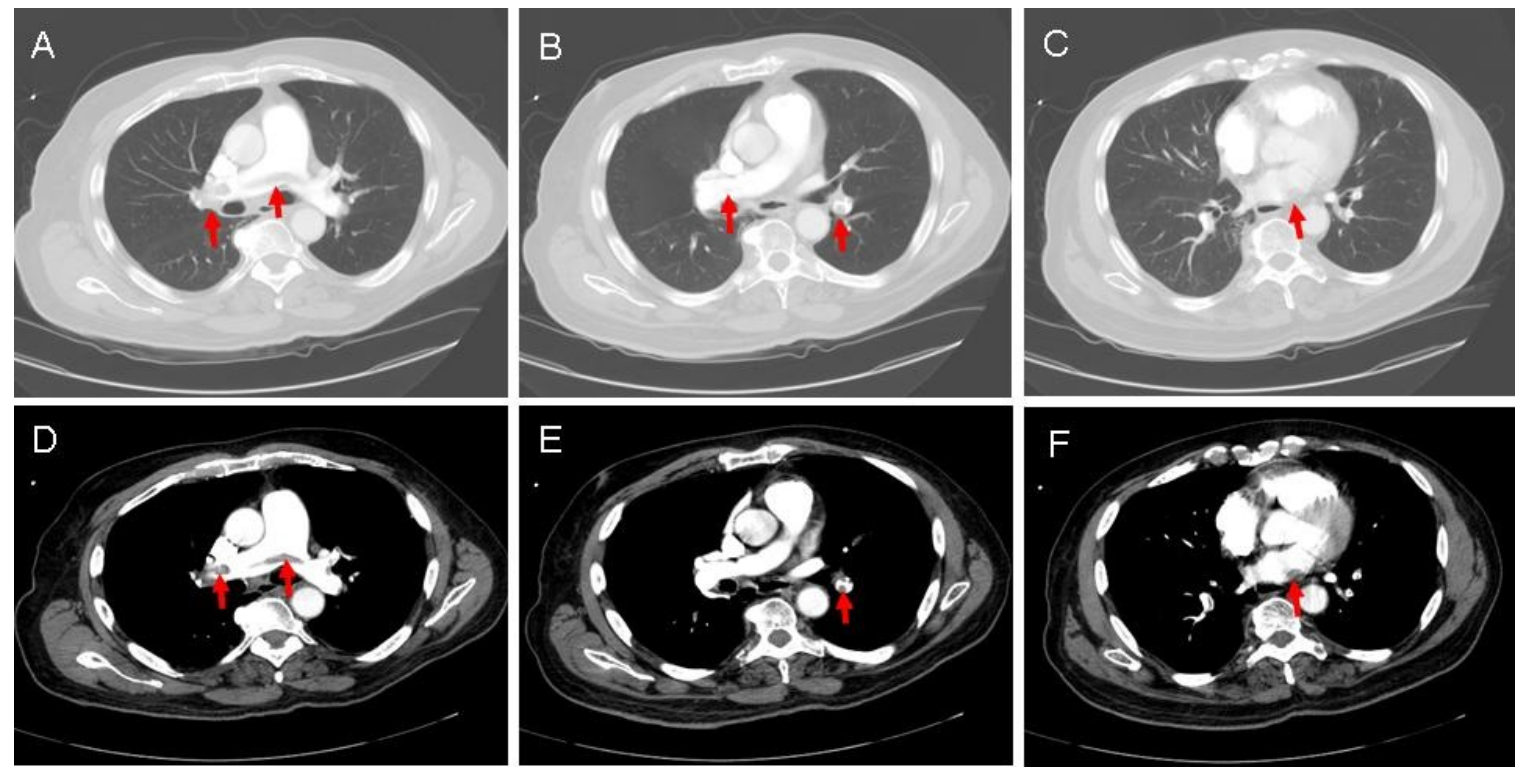

Figure 3. Intraluminal filling defects (red arrows).

The diagnosis is not surprising. A patient who becomes dyspneic, tachycardic and hypoxic post-operatively with a normal chest x-ray is at high risk for pulmonary embolism.

Which of the following is indicated at this time?

1. Brain natriuretic peptide (BNP)

2. Echocardiography

3. Troponin

4. All of the above

5. None of the above 


\section{Correct! \\ 4. All of the above}

Studies have suggested that right ventricular dysfunction with massive or submassive pulmonary embolism predicts a higher mortality rate (1). Echocardiography as well as the cardiac biomarkers BNP and troponin have been used to detect right ventricular dysfunction. In this patient's case the echocardiography showed a large mobile density in the pulmonary artery consistent with a pulmonary embolism. In addition, there was a flattened septum with the right ventricle moderately dilated with moderate systolic dysfunction. The troponin and BNP were modestly elevated at $0.3 \mathrm{mcg} / \mathrm{L}$ and $477 \mathrm{pg} / \mathrm{ml}$.

Which of the following would be appropriate management decisions?

1. Catheter-directed embolectomy

2. Catheter-directed thrombolysis

3. Intravenous heparin

4. Low dose thrombolytics

5. Ultrasound-induced thrombolysis 


\section{Correct! \\ 1. Catheter-directed embolectomy \\ 2. Catheter-directed thrombolysis \\ 3. Intravenous heparin \\ 4. Low dose thrombolytics \\ 5. Ultrasound-induced thrombolysis}

At this juncture the optimal initial therapy for massive or submassive pulmonary embolism in the absence of hypotension is unclear and therefore anticoagulation with or without thrombolytics is appropriate (2). In this case catheter-directed administration of tissue plasminogen activator (tPA) and ultrasound-induced thrombolysis was performed with the EKOS catheter. The results showed a dramatic thrombolysis of the embolism (Figure 4).

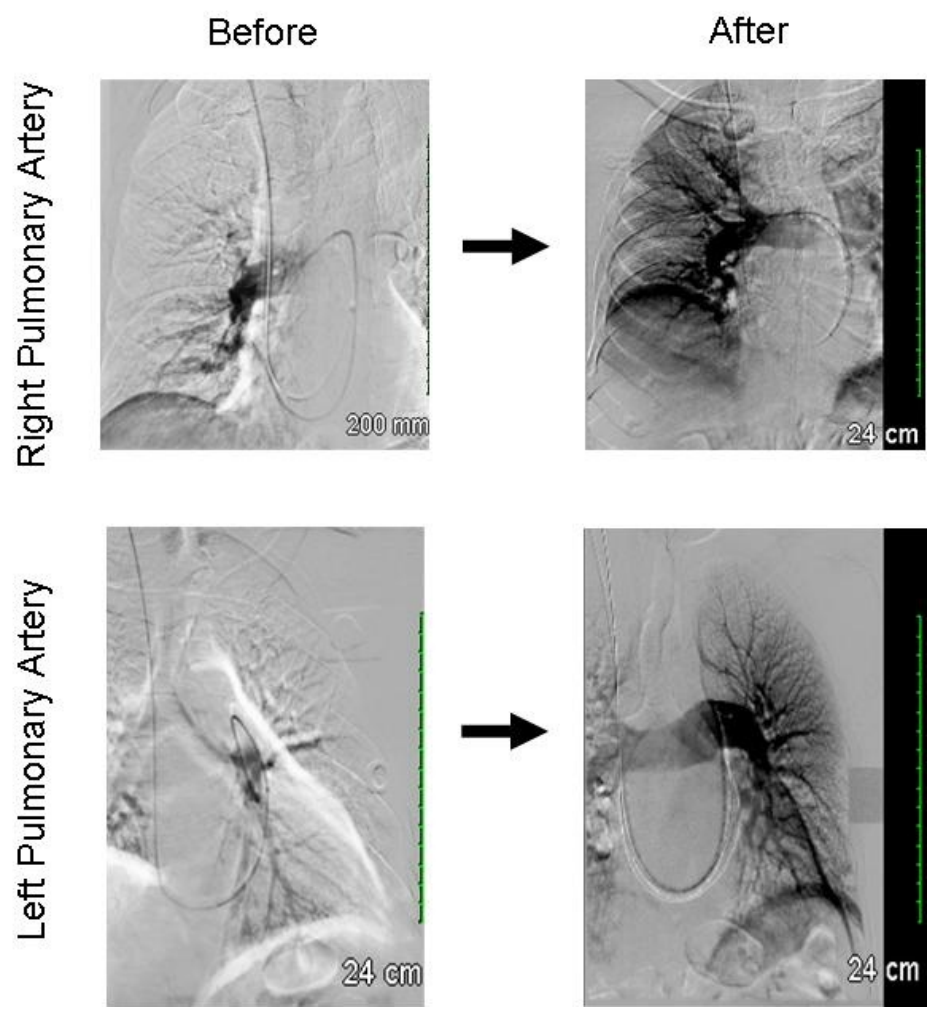

Figure 4. Pulmonary angiography before and after thrombolysis.

\section{Catheter Directed Thrombolysis}

The use of catheters to directly infuse thrombolytics near or within a thrombus is not a new technology; there are case reports of this technology as far back as the 1970's. However, it has only been recently that these techniques have been more widely adopted. 
The level of evidence supporting the use of catheter directed thrombolysis is very low. There is only one randomized controlled trial that compared intravenous to intrapulmonary thrombolytic administration (3). This study was published in 1988 and included 34 patients with massive acute pulmonary embolism. No difference was found in any outcome, other than patients undergoing catheter directed thrombolysis experienced a higher risk of bleeding at the catheter insertion site.

\section{Further Development of Catheter-based Technology}

Undeterred by the 1988 trial, interventional radiologists have continued to develop more advanced technologies; the majority of these technologies are used in conjunction with catheter directed thrombolysis, but they can also be used in isolation in patients with absolute contraindications to thrombolytics $(4,5)$.

Thrombus fragmentation by rotating a pigtail catheter is the most straightforward and least advanced of these methods. While good success can be achieved in disrupting occlusive thrombi, there is also a very high risk of macroembolization of the macerated clot, which could result in further hemodynamic deterioration; this method is not frequently used anymore.

Rheolytic thrombectomy utilizes catheters with a high-pressure saline jet within the catheter; this creates a gradient along which the thrombus is suctioned into the catheter. Other catheters utilize a high-speed coil (rotational thrombectomy) to create a negative pressure gradient within the catheter; the coil also facilitates maceration of the thrombus. Both rheolytic thrombectomy and rotational thrombectomy can be complicated by bradycardia, heart block, and asystole.

\section{Ultrasound Enhanced Thrombolysis- the EKOS Catheter}

The concept of using ultrasound waves to disrupt clots is not novel; in vitro models demonstrated its potential for clinical usefulness in the early 1990's. Animal models were developed shortly thereafter. By the late 1990's, the first endovascular prototype was developed. In 2004, EKOS gained approval for the use of this system in humans.

The data supporting the use of this device is very slim; there are no randomized controlled trials currently published comparing the EKOS catheter to systemic thrombolytics. There are several small trials in process; however, these studies

are using surrogate endpoints (improvement in RV enlargement at 24 hours) and will likely have uncertain clinical significance.

\section{Low Dose Thrombolytics}

One major barrier to administration of systemic thrombolytics to patients with anything other than hemodynamic compromise is the concern of bleeding. This concern combined with the observation that a significant proportion of patients 
with a large clot burden will develop chronic thromboembolic pulmonary hypertension led a team of investigators (MOPETT) to study the efficacy of lowdose thrombolytics on the incidence of pulmonary hypertension in patients with "moderate" PE (6). In contrast to the full dose $100 \mathrm{mg}$ tPA that is typically administered, patients in the treatment arm of this trial received $50 \mathrm{mg}$ of tPA (administered as a $10 \mathrm{mg}$ bolus followed by $40 \mathrm{mg}$ over $2 \mathrm{hrs}$ ). All patients received heparin; they were then randomized to either anticoagulation only or to low dose thrombolytic therapy plus continued anticoagulation with heparin. The primary outcome was evidence of pulmonary hypertension $(\mathrm{PH})$ at 28 months as assessed by echocardiography. PH was present in $57 \%$ of patients treated with anticoagulation only and in $16 \%$ of those treated with thrombolytics + anticoagulation. There were no bleeding complications in either group.

\section{References}

1. Konstantinides S. Pulmonary embolism: impact of right ventricular dysfunction. Curr Opin Cardiol. 2005;20(6):496-501. [CrossRef] [PubMed]

2. Kearon C, Akl EA, Comerota AJ, Prandoni P, Bounameaux H, Goldhaber SZ, Nelson ME, Wells PS, Gould MK, Dentali F, Crowther M, Kahn SR; American College of Chest Physicians. Antithrombotic therapy for VTE disease:

Antithrombotic Therapy and Prevention of Thrombosis, 9th ed: American College of Chest Physicians Evidence-Based Clinical Practice Guidelines. Chest. 2012;141:e419S-94S. [CrossRef] [PubMed]

3. Verstraete M, Miller GA, Bounameaux H, Charbonnier B, Colle JP, Lecorf G, Marbet GA, Mombaerts P, Olsson CG. Intravenous and intrapulmonary recombinant tissue-type plasminogen activator in the treatment of acute massive pulmonary embolism. Circulation. 1988;77(2):353-60. [CrossRef] [PubMed]

4. Cuculi F, Kobza R, Bergner M, Erne P. Usefulness of aspiration of pulmonary emboli and prolonged local thrombolysis to treat pulmonary embolism. Am J Cardiol. 2012; 110: 1841-1845. [CrossRef] [PubMed]

5. Kuo WT. Endovascular therapy for acute pulmonary embolism. J Vasc Interv Radiol. 2012; 23: 167-179. [CrossRef] [PubMed]

6. Sharifi M, Bay C, Skrocki L, Rahimi F, Mehdipour M; "MOPETT" Investigators. Moderate pulmonary embolism treated with thrombolysis (from the "MOPETT" trial). Am J Cardiol. 2013; 111: 273-277. [CrossRef] [PubMed] 